Journal of Applied ANALysis

Vol. 7, No. 1 (2001), pp. 91-105

\title{
SOME MODIFICATIONS OF DENSITY TOPOLOGIES
}

\section{G. HORBACZEWSKA and E. WAGNER-BOJAKOWSKA}

Received February 29, 2000 and, in revised form, September 5, 2000

\begin{abstract}
In this paper we introduce two topologies on the plane connected with the notions of density and $I$-density. Their definitions are based on the notion of a regular density point. We investigate connections between them and the density and $I$-density topologies on the plane and on the real line. We consider axioms of separation and functions continuous with respect to these topologies.
\end{abstract}

\section{Introduction}

The aim of this paper is to introduce some topologies connected with the notions of density and $I$-density. The motivation to consider such topologies comes from the notion of a regular approximate differential ([2, 408-409]), the definition of which is based on the notion of a regular density point.

For terminology and definitions concerning density points and the density topologies on the real line and on the plane, see [4]. Information on $I$-density points and the $I$-density topologies on the real line and on the plane are contained in [7], [8], [9], [1], [3].

1991 Mathematics Subject Classification. 54 A 10, 54 C 05, 28 A 05. point.

Key words and phrases. Density topologies, comparison of topologies, regular density

ISSN 1425-6908 C Heldermann Verlag. 


\section{The measure density case}

Let $\mathbb{R}$ denote the real line, $\mathbb{R}^{2}$ - the plane, $\mathbb{R}_{+}$- the set of positive real numbers, $\mathbb{Q}$ - the set of rational numbers, $\mathbb{N}$ - the set of positive integers.

Denote by $m_{k}$ the Lebesgue measure and by $\mathcal{L}_{k}$ the family of all Lebesgue measurable sets on $\mathbb{R}^{k}, k=1,2$. Let $d_{k}(A, p)\left(\underline{d_{k}}(A, p)\right)$ denote the ordinary density (lower ordinary density) of a measurable set $A \subset \mathbb{R}^{k}$ at a point $p \in \mathbb{R}^{k}, k=1,2$. If a plane set is contained in a line, then we shall consider its linear measure and we use the linear density $d_{1}$.

Let $A^{\prime}$ denote the complement of $A$. If $A \subset \mathbb{R}$ then $A^{\prime}=\mathbb{R} \backslash A$. If $A \subset \mathbb{R}$ and $x_{0} \in \mathbb{R}$ then $A+x_{0}=\left\{a+x_{0}: a \in A\right\}$ and $-A=\{-a: a \in A\}$.

Let $\left(x_{0}, y_{0}\right) \in \mathbb{R}^{2}, t \in \mathbb{R}_{+}$. Then we denote

$R\left(\left(x_{0}, y_{0}\right), t\right)=\left(\left[x_{0}-t, x_{0}+t\right] \times\left[y_{0}-t, y_{0}+t\right]\right) \backslash\left(\left(x_{0}-t, x_{0}+t\right) \times\left(y_{0}-t, y_{0}+t\right)\right)$ and

$$
\mathcal{R}\left(x_{0}, y_{0}\right)=\left\{B \subset \mathbb{R}^{2}: B=\bigcup_{t \in T} R\left(\left(x_{0}, y_{0}\right), t\right) \quad \text { for some } \quad T \subset \mathbb{R}_{+}\right\} .
$$

If $x_{0}=y_{0}=0$, we write $R(t)$ instead of $R\left(\left(x_{0}, y_{0}\right), t\right)$, and $\mathcal{R}$ instead of $\mathcal{R}\left(x_{0}, y_{0}\right)$.

If $A \subset \mathbb{R}^{2}$ then let

$$
A_{[R]}^{+}\left(x_{0}, y_{0}\right)=\left\{t \in \mathbb{R}_{+}: R\left(\left(x_{0}, y_{0}\right), t\right) \subset A\right\}
$$

and

$$
A_{[R]}\left(x_{0}, y_{0}\right)=A_{[R]}^{+}\left(x_{0}, y_{0}\right) \cup\left(-A_{[R]}^{+}\left(x_{0}, y_{0}\right)\right) .
$$

Obviously, $A_{[R]}\left(x_{0}, y_{0}\right)=-A_{[R]}\left(x_{0}, y_{0}\right)$. If $x_{0}=y_{0}=0$, we write $A_{[R]}$ instead of $A_{[R]}\left(x_{0}, y_{0}\right)$. If $A \subset \mathbb{R}^{2}$ and $x \in \mathbb{R}$ then $A_{x}=\{y \in \mathbb{R}:(x, y) \in A\}$.

Let $\mathcal{O}$ denote the Euclidean topology on the plane. If $\mathcal{T}$ is an arbitrary topology on the plane then by $C_{\mathcal{T}}$ we shall denote the family of all functions $f: \mathbb{R}^{2} \rightarrow \mathbb{R}$ continuous with respect to the topology $\mathcal{T}$ on the domain and the Euclidean topology on the range.

We shall say that two sets $A, B \subset \mathbb{R}^{2}$ are equivalent $(A \sim B)$ if and only if $m_{2}(A \triangle B)=0$ where $A \triangle B=(A \backslash B) \cup(B \backslash A)$.

Let $\mathcal{D}_{1}\left(\mathcal{D}_{2}\right)$ denote the density topology on the real line (the ordinary density topology on the plane).

A function $f$ is called Baire* 1 ([5]) if for every perfect set $P$ there exists a portion $Q$ of $P$ (that is a nonempty set of the form $U \cap P$ where $U$ is a nondegenerate interval) such that $f \mid Q$ is continuous.

Definition 2.1. We say that a point $\left(x_{0}, y_{0}\right) \in \mathbb{R}^{2}$ is a regular density point of a set $A \subset \mathbb{R}^{2}$ if and only if there exists a measurable set $B \subset A$ such that $d_{2}\left(B,\left(x_{0}, y_{0}\right)\right)=1$ and $B \in \mathcal{R}\left(x_{0}, y_{0}\right)$. In this case we write $d_{r}\left(A,\left(x_{0}, y_{0}\right)\right)=1$. 
For $A \in \mathcal{L}_{2}$ let

$$
\Phi(A)=\left\{(x, y) \in \mathbb{R}^{2}: d_{2}(A,(x, y))=1\right\}
$$

and

$$
\Phi_{r}(A)=\left\{(x, y) \in \mathbb{R}^{2}: d_{r}(A,(x, y))=1\right\} .
$$

Obviously, $\Phi_{r}(A) \subset \Phi(A)$ for $A \in \mathcal{L}_{2}$. Observe that the operator $\Phi_{r}$ has the following properties:

$1^{0} \quad \Phi_{r}(\emptyset)=\emptyset, \Phi_{r}\left(\mathbb{R}^{2}\right)=\mathbb{R}^{2} ;$

$2^{0}$ if $A \subset B$, then $\Phi_{r}(A) \subset \Phi_{r}(B)$;

$3^{0} \quad \Phi_{r}(A \cap B)=\Phi_{r}(A) \cap \Phi_{r}(B)$

for $A, B \in \mathcal{L}_{2}$. It suffices to show that

$$
\Phi_{r}(A) \cap \Phi_{r}(B) \subset \Phi_{r}(A \cap B)
$$

(the remaining properties follow immediately from the definition of $\Phi_{r}$ ). Let $\left(x_{0}, y_{0}\right) \in \Phi_{r}(A) \cap \Phi_{r}(B)$. Then there exist two sets $C, D \in \mathcal{L}_{2} \cap \mathcal{R}\left(x_{0}, y_{0}\right)$ such that $C \subset A, D \subset B, d_{2}\left(C,\left(x_{0}, y_{0}\right)\right)=d_{2}\left(D,\left(x_{0}, y_{0}\right)\right)=1$. Obviously, $C \cap D \subset A \cap B, C \cap D \in \mathcal{R}\left(x_{0}, y_{0}\right)$ and $d_{2}\left(C \cap D,\left(x_{0}, y_{0}\right)\right)=1$ since $\Phi(C) \cap \Phi(D)=\Phi(C \cap D)$ (see [6, Theorem 22.4]). Consequently, $\left(x_{0}, y_{0}\right) \in$ $\Phi_{r}(A \cap B)$.

Observe that $\Phi_{r}$ is not a so called "lower density operator" (see [6, Theorem 22.4]) since the Lebesgue Density Theorem does not hold for $\Phi_{r}$. Indeed, let

$$
A=[-1,1] \times((\mathbb{R} \backslash \mathbb{Q}) \cap[-1,1]) .
$$

Then $m_{2}(A)=4$ and $\Phi_{r}(A)=\emptyset$, so $m_{2}\left(A \triangle \Phi_{r}(A)\right)=4$.

We require the "lower density" has the same value for equivalent sets, but our operator has not this property. There exist two measurable sets $C, D \subset \mathbb{R}^{2}$ (namely, $C=[-1,1]^{2}, D=A$ ) such that $C \sim D$ (that is $\left.m_{2}(C \triangle D)=0\right)$ and $\Phi_{r}(C)=(-1,1)^{2} \neq \Phi_{r}(D)$.

Put

$$
\mathcal{T}_{r}=\left\{A \in \mathcal{L}_{2}: A \subset \Phi_{r}(A)\right\}
$$

Theorem 2.2. The family $\mathcal{T}_{r}$ is a topology on the plane, essentially stronger than the Euclidean topology and essentially weaker than the density topology $\mathcal{D}_{2}$ on the plane.

Proof. Obviously, $\emptyset, \mathbb{R}^{2} \in \mathcal{T}_{r}$. By property $3^{0}$ the family $\mathcal{T}_{r}$ is closed under finite intersections. It suffices to show that $\mathcal{T}_{r}$ is closed also under arbitrary unions. Let $\left\{A_{\alpha}, \alpha \in \Lambda\right\}$ be a subfamily of $\mathcal{T}_{r}$. Then $A_{\alpha} \subset \Phi_{r}\left(A_{\alpha}\right)$ for $\alpha \in \Lambda$. 
We first prove that $\bigcup_{\alpha \in \Lambda} A_{\alpha} \in \mathcal{L}_{2}$. We have $A_{\alpha} \subset \Phi_{r}\left(A_{\alpha}\right) \subset \Phi\left(A_{\alpha}\right)$ and $A_{\alpha} \in \mathcal{L}_{2}$, so $A_{\alpha}$ is open in the density topology for $\alpha \in \Lambda$. Hence $\bigcup_{\alpha \in \Lambda} A_{\alpha}$ is also open in the density topology and, consequently, it is a measurable set.

Now, we observe that $\bigcup_{\alpha \in \Lambda} A_{\alpha} \subset \bigcup_{\alpha \in \Lambda} \Phi_{r}\left(A_{\alpha}\right) \subset \Phi_{r}\left(\bigcup_{\alpha \in \Lambda} A_{\alpha}\right)$ by monotonicity of $\Phi_{r}$ and by the inclusion $A_{\alpha} \subset \Phi_{r}\left(A_{\alpha}\right)$ for $\alpha \in \Lambda$.

The inclusions $\mathcal{O} \subset \mathcal{T}_{r} \subset \mathcal{D}_{2}$ are obvious. Put

$$
A=[(\mathbb{R} \backslash \mathbb{Q}) \times \mathbb{R}] \cup[\mathbb{R} \times(\mathbb{R} \backslash \mathbb{Q})] \text { and } B=(\mathbb{R} \backslash \mathbb{Q}) \times \mathbb{R} .
$$

Then $A \in \mathcal{T}_{r} \backslash \mathcal{O}$, since $\mathbb{R}^{2} \backslash A \subset \mathbb{Q} \times \mathbb{Q}$, and $B \in \mathcal{D}_{2} \backslash \mathcal{T}_{r}$, since $\mathbb{R}^{2} \backslash B=$ $\mathbb{Q} \times \mathbb{R}$.

Now we indicate connections between the regular density and the linear density.

Let $p_{x_{0}}$ denote the line $x=x_{0}$ and $p^{y_{0}}$ - the line $y=y_{0}$.

Lemma 2.3. Let $B \in \mathcal{R}$. The set $B$ is measurable $\left(B \in \mathcal{L}_{2}\right)$ if and only if $B_{[R]} \in \mathcal{L}_{1}$.

Proof. Let $B \in \mathcal{L}_{2}$ and $B \in \mathcal{R}$. Put

$$
Z=\left\{t \in \mathbb{R}_{+}: B_{t} \notin \mathcal{L}_{1}\right\} .
$$

By the Fubini theorem $m_{1}(Z)=0$. Let $\left\{t_{n}\right\}_{n \in \mathbb{N}}$ be a decreasing sequence of numbers from $\mathbb{R}_{+} \backslash Z$ tending to zero. Then $B_{t_{n}} \in \mathcal{L}_{1}$ for $n \in \mathbb{N}$, so $B_{t_{n}} \cap\left[t_{n}, \infty\right) \in \mathcal{L}_{1}$ and

$$
B_{[R]}^{+}=\bigcup_{n \in \mathbb{N}}\left(B_{t_{n}} \cap\left[t_{n}, \infty\right)\right) .
$$

Consequently, $B_{[R]}^{+}$and also $B_{[R]}$ are measurable sets on the real line.

Suppose now that $B_{[R]} \in \mathcal{L}_{1}$. Obviously, $B_{[R]} \times \mathbb{R}, \mathbb{R} \times B_{[R]} \in \mathcal{L}_{2}$. Put

$$
C_{I}=\left\{(x, y) \in \mathbb{R}^{2}:|y| \leq|x|\right\}
$$

and

$$
C_{I I}=\left\{(x, y) \in \mathbb{R}^{2}:|x| \leq|y|\right\} .
$$

Clearly, $C_{I}, C_{I I} \in \mathcal{L}_{2}$ and

$$
B=\left[\left(B_{[R]} \times \mathbb{R}\right) \cap C_{I}\right] \cup\left[\left(\mathbb{R} \times B_{[R]}\right) \cap C_{I I}\right] .
$$

Consequently, $B \in \mathcal{L}_{2}$.

Definition 2.4. We say that $x \in \mathbb{R}$ is an inner density point of $A \subset \mathbb{R}$ if and only if there exists a set $B \in \mathcal{L}_{1}$ such that $B \subset A$ and $d_{1}(B, x)=1$. 
Theorem 2.5. If $(0,0)$ is a regular density point of a set $A \subset \mathbb{R}^{2}$ then $(0,0)$ is an inner (linear) density point of $A \cap p_{0}$ and $A \cap p^{0}$.

Proof. We shall show that if $d_{r}(A,(0,0))=1$ then $(0,0)$ is an inner density point of $A \cap p_{0}$.

By assumption there exists a measurable set $B \subset A, B \in \mathcal{R}$, such that $d_{2}(B,(0,0))=1$. It is sufficient to prove that $d_{1}\left(B_{[R]}, 0\right)=1$ since $B_{[R]} \times\{0\} \subset A \cap p_{0}$.

Suppose, contrary to our claim, that $\underline{d}_{1}\left(B_{[R]}, 0\right)<1$. So there exist a positive number $\varepsilon$ and a decreasing sequence $\left\{t_{n}\right\}_{n \in \mathbb{N}}$ of positive real numbers tending to zero such that

$$
\lim _{n \rightarrow \infty} \frac{m_{1}\left(B_{[R]} \cap\left[-t_{n}, t_{n}\right]\right)}{2 t_{n}}<1-\varepsilon .
$$

Changing the numeration, if necessary, we may assume that for every $n \in \mathbb{N}$

$$
m_{1}\left(B_{[R]} \cap\left[-t_{n}, t_{n}\right]\right)<(1-\varepsilon) 2 t_{n} .
$$

By the symmetry of $B_{[R]}\left(B_{[R]}=-B_{[R]}\right)$ we have

$$
m_{1}\left(B_{[R]} \cap\left[0, t_{n}\right]\right)<(1-\varepsilon) t_{n},
$$

so

$$
m_{1}\left(\left(B_{[R]}\right)^{\prime} \cap\left[0, t_{n}\right]\right) \geq \varepsilon t_{n} .
$$

Put

$$
C_{n}=\left\{(x, y) \in \mathbb{R}^{2}: 0 \leq y \leq x \quad \text { and } \quad x \in\left[0, t_{n}\right]\right\}
$$

Thus

Consequently,

$$
m_{2}\left(B^{\prime} \cap C_{n}\right) \geq \frac{\varepsilon^{2} t_{n}^{2}}{2}
$$

$$
m_{2}\left(B \cap C_{n}\right) \leq m_{2}\left(C_{n}\right)-\frac{\varepsilon^{2} t_{n}^{2}}{2}=\frac{t_{n}^{2}\left(1-\varepsilon^{2}\right)}{2},
$$

so

$$
\frac{m_{2}\left(B \cap\left[-t_{n}, t_{n}\right]^{2}\right)}{4 t_{n}^{2}}=\frac{8 m_{2}\left(B \cap C_{n}\right)}{4 t_{n}^{2}} \leq 1-\varepsilon^{2}<1 .
$$

Therefore

$$
\liminf _{t \rightarrow 0^{+}} \frac{m_{2}\left(B \cap[-t, t]^{2}\right)}{4 t^{2}} \leq \liminf _{n \rightarrow \infty} \frac{m_{2}\left(B \cap\left[-t_{n}, t_{n}\right]^{2}\right)}{4 t_{n}^{2}}<1,
$$

a contradiction.

The analogous considerations can be carried out for $A \cap p^{0}$.

Theorem 2.6. Let $B \in \mathcal{R}$. If $d_{1}\left(B_{[R]}, 0\right)=1$ then $d_{2}(B,(0,0))=1$. 
Proof. From our assumption we have

$$
\lim _{h \rightarrow 0^{+}} \frac{m_{1}\left(B_{[R]} \cap[0, h]\right)}{h}=1,
$$

so for each $\varepsilon>0$ there exists a positive number $\delta$ such that

$$
\frac{m_{1}\left(B_{[R]} \cap[0, h]\right)}{h}>1-\varepsilon
$$

for $h \in(0, \delta)$. Therefore

$$
m_{1}\left(\left(B_{[R]}\right)^{\prime} \cap[0, h]\right)<\varepsilon h
$$

for $h \in(0, \delta)$. Put

$$
C_{h}=\left\{(x, y) \in \mathbb{R}^{2}: 0 \leq y \leq x \quad \text { and } \quad x \in[0, h]\right\} .
$$

Then

Hence

$$
m_{2}\left(B^{\prime} \cap C_{h}\right)<\frac{1}{2}(h+h-\varepsilon h) \varepsilon h=\frac{1}{2}(2-\varepsilon) \varepsilon h^{2} .
$$

for $h \in(0, \delta)$. Consequently,

$$
\frac{m_{2}\left(B^{\prime} \cap[-h, h]^{2}\right)}{4 h^{2}}<(2-\varepsilon) \varepsilon<2 \varepsilon
$$

$$
\lim _{h \rightarrow 0^{+}} \frac{m_{2}\left(B^{\prime} \cap[-h, h]^{2}\right)}{4 h^{2}}=0,
$$

so $d_{2}(B,(0,0))=1$.

Corollary 2.7. If $B \in \mathcal{R}$ then the following conditions are equivalent:

a) $d_{1}\left(B_{[R]}, 0\right)=1$,

b) $d_{2}(B,(0,0))=1$,

c) $d_{r}(B,(0,0))=1$.

Of course, the same results can be obtained for any point $\left(x_{0}, y_{0}\right)$ taken instead of $(0,0)$.

Let $\mathcal{D} \times \mathcal{D}$ denote the product density topology on the plane.

Theorem 2.8. $\mathcal{T}_{r} \varsubsetneqq \mathcal{D} \times \mathcal{D}$.

Proof. Let $A \in \mathcal{T}_{r}$ and $\left(x_{0}, y_{0}\right) \in A$. There exists a measurable set $B \subset A$ such that $B \in \mathcal{R}\left(x_{0}, y_{0}\right)$ and $d_{2}\left(B,\left(x_{0}, y_{0}\right)\right)=1$. From Theorem 2.5 it follows that

and

$$
d_{1}\left(B_{[R]}\left(x_{0}, y_{0}\right)+x_{0}, x_{0}\right)=1
$$

$$
d_{1}\left(B_{[R]}\left(x_{0}, y_{0}\right)+y_{0}, y_{0}\right)=1 .
$$

Simultaneously, $\left(B_{[R]}\left(x_{0}, y_{0}\right)+x_{0}\right) \times\left(B_{[R]}\left(x_{0}, y_{0}\right)+y_{0}\right) \subset A$, so $\left(x_{0}, y_{0}\right)$ is the interior point of $A$ in the topology $\mathcal{D} \times \mathcal{D}$. From the arbitrariness of $\left(x_{0}, y_{0}\right)$ 
it follows that $A \in \mathcal{D} \times \mathcal{D}$. Put $B=(\mathbb{R} \backslash \mathbb{Q}) \times \mathbb{R}$. Obviously, $B \in \mathcal{D} \times \mathcal{D}$. Simultaneously, $\Phi_{r}(B)=\emptyset$, so $B \notin \mathcal{T}_{r}$.

Theorem 2.9. Let $A \in \mathcal{L}_{2}$. If $m_{1}\left(\operatorname{proj}_{y} A\right)=0$ and $m_{1}\left(\operatorname{proj}_{x} A\right)=0$ then $A$ is closed in the topology $\mathcal{T}_{r}$.

Proof. Let $\left(x_{0}, y_{0}\right) \in A^{\prime}$. Assume first that $\left(x_{0}, y_{0}\right)=(0,0)$. Put

$$
Z_{1}=\operatorname{proj}_{x} A \cup \operatorname{proj}_{y} A
$$

and

$$
Z=Z_{1} \cup\left(-Z_{1}\right)
$$

Obviously, $Z$ is symmetric with respect to zero and $m_{1}(Z)=0$. Note that if $t \notin Z$ then

$$
t \notin \operatorname{proj}_{x} A \cup \operatorname{proj}_{y} A \cup\left(-\operatorname{proj}_{x} A\right) \cup\left(-\operatorname{proj}_{y} A\right),
$$

so

$$
p_{t} \cap A=\emptyset, \quad p_{-t} \cap A=\emptyset, \quad p^{t} \cap A=\emptyset \text { and } p^{-t} \cap A=\emptyset .
$$

Therefore $R(t) \subset A^{\prime}$ for $t \in Z^{\prime} \cap \mathbb{R}_{+}$, hence $\bigcup_{t \in Z^{\prime} \cap \mathbb{R}_{+}} R(t) \subset A^{\prime}$. Put

$$
B=\bigcup_{t \in Z^{\prime} \cap \mathbb{R}_{+}} R(t)
$$

Clearly, $B_{[R]}=Z^{\prime}$, so $d_{1}\left(B_{[R]}, 0\right)=1$. By Corollary 2.7 we have $d_{2}(B,(0,0))=1$ and, consequently, $d_{r}\left(A^{\prime},(0,0)\right)=1$.

Now let $\left(x_{0}, y_{0}\right) \neq(0,0)$. Put

$$
Z=\operatorname{proj}_{x} A \cup\left(2 x_{0}-\operatorname{proj}_{x} A\right) \cup\left(x_{0}-y_{0}+\operatorname{proj}_{y} A\right) \cup\left(y_{0}+x_{0}-\operatorname{proj}_{y} A\right) \text {. }
$$

Obviously, $m_{1}(Z)=0$. If $t \notin Z$ then

$t \notin \operatorname{proj}_{x} A, t \notin 2 x_{0}-\operatorname{proj}_{x} A, t \notin x_{0}-y_{0}+\operatorname{proj}_{y} A, t \notin y_{0}+x_{0}-\operatorname{proj}_{y} A$, hence

$$
p_{t} \cap A=\emptyset, p_{2 x_{0}-t} \cap A=\emptyset, p^{y_{0}-x_{0}+t} \cap A=\emptyset, p^{y_{0}+x_{0}-t} \cap A=\emptyset .
$$

Therefore $R\left(\left(x_{0}, y_{0}\right), t\right) \subset A^{\prime}$ for $t \in Z^{\prime} \cap \mathbb{R}_{+}$. Put

$$
B=\bigcup_{t \in Z^{\prime} \cap \mathbb{R}_{+}} R\left(\left(x_{0}, y_{0}\right), t\right) .
$$

Clearly, $B \subset A^{\prime}$ and $B_{[R]}\left(x_{0}, y_{0}\right)=Z^{\prime}$, so $d_{1}\left(B_{[R]}\left(x_{0}, y_{0}\right), 0\right)=1$. By Corollary 2.7 (applied to the set $B$ moved by the vector $\left[-x_{0},-y_{0}\right]$ ) we have $d_{2}\left(B,\left(x_{0}, y_{0}\right)\right)=1$ and, consequently, $d_{r}\left(A^{\prime},\left(x_{0}, y_{0}\right)\right)=1$. From the arbitrariness of $\left(x_{0}, y_{0}\right) \in A^{\prime}$ it follows that $A^{\prime} \in \mathcal{T}_{r}$.

Corollary 2.10. The space $\left(\mathbb{R}^{2}, \mathcal{T}_{r}\right)$ is not separable. 
Corollary 2.11. The space $\left(\mathbb{R}^{2}, \mathcal{T}_{r}\right)$ is not compact.

Proof. The $\mathcal{T}_{r}$-compact sets are the finite sets. If $E$ is infinite, then it contains a countable infinite subset $E_{1}$, which is not compact in the Euclidean topology. If $E$ is $\mathcal{T}_{r}$-compact then $E_{1}$ would be also $\mathcal{T}_{r}$ - compact which gives a contradiction.

It is natural to investigate the separation axioms for the topology $\mathcal{T}_{r}$. Obviously, $\mathcal{T}_{r}$ is stronger than the Euclidean topology on the plane, so it is a Hausdorff $\left(T_{2}\right)$ topology.

Observe, that $\mathcal{T}_{r}$ is not normal. Indeed, let $L$ and $R$ denote the sets of left and right end points of component intervals of the complement of Cantor set $C \subset \mathbb{R}$, respectively. The sets $L$ and $R$ are countable, so they are closed in $\mathcal{T}_{r}$ and $L \cap R=\emptyset$. Suppose that $\left(\mathbb{R}^{2}, \mathcal{T}_{r}\right)$ is a normal $\left(T_{4}\right)$ space. Then there exists a continuous function $f:\left(\mathbb{R}^{2}, \mathcal{T}_{r}\right) \rightarrow([0,1], \mathcal{O})$ such that $f(L \times\{0\})=\{1\}$ and $f(R \times\{0\})=\{0\}$. Clearly, each point of $C$ is an accumulation point of $R$ and $L$, so $f$ is discontinuous everywhere on $C \times\{0\}$ as a function from the plane with the Euclidean topology. Simultaneously, $\mathcal{T}_{r} \subset \mathcal{D}_{2}$ and, consequently, each function continuous with respect to $\mathcal{T}_{r}$ on the domain is in the first class of Baire - a contradiction.

Now we shall prove that the family of all functions continuous with respect to $\mathcal{T}_{r}$ on the domain is not contained in the class of Baire* 1 functions introduced by O'Malley ([5]).

We say that any of the sets $\bigcup_{n \in \mathbb{N}}\left(a_{n}, b_{n}\right)$ or $\bigcup_{n \in \mathbb{N}}\left[a_{n}, b_{n}\right]$ is a right (left) interval set at a point $x \in \mathbb{R}$ if $a_{n+1}<b_{n+1}<a_{n}<b_{n}\left(a_{n+1}>b_{n+1}>a_{n}>\right.$ $b_{n}$ ) for $n \in \mathbb{N}$ and $\lim _{n \rightarrow \infty} a_{n}=x$. We say that a set $E \subset \mathbb{R}$ is an interval set at a point $x \in \mathbb{R}$ if it is a union of a right interval set and a left interval set at $x$.

For our purpose we shall use Lemma 2.1.8 from [3] in the following form:

Lemma 2.12. There exists a perfect set $C \subset \mathbb{R}$ such that for every $x \in C$ there is an interval set $E$ at $x$ such that $C \subset E \cup\{x\}$ and $d_{1}(E, x)=0$.

Theorem 2.13. There exists a function $f: \mathbb{R}^{2} \rightarrow \mathbb{R}$ continuous with respect to the topology $\mathcal{T}_{r}$ on the domain, which is not in Baire* 1 class.

Proof. Let us consider the set $C$ from Lemma 2.12. We may assume that $C \subset[0,1]$. Let

$$
[0,1] \backslash C=\bigcup_{k=1}^{\infty}\left(a_{k}, b_{k}\right)
$$


Clearly, $a_{1} \in C$. By Lemma 2.12 there exists an interval set $E_{1} \subset$ $\left[a_{1}-\left(b_{1}-a_{1}\right) / 2, a_{1}+\left(b_{1}-a_{1}\right) / 2\right]$ symmetric with respect to $a_{1}$, which is a union of open intervals such that

$$
C \cap\left[a_{1}-\frac{b_{1}-a_{1}}{2}, a_{1}+\frac{b_{1}-a_{1}}{2}\right] \subset E_{1} \cup\left\{a_{1}\right\}
$$

and

$$
d_{1}\left(E_{1}, a_{1}\right)=0
$$

Put

$$
H_{1}=\left[a_{1}-\frac{b_{1}-a_{1}}{2}, a_{1}+\frac{b_{1}-a_{1}}{2}\right] \backslash\left(E_{1} \cup\left\{a_{1}\right\}\right) .
$$

Obviously, $H_{1}$ is an interval set at the point $a_{1}$, which is a union of closed intervals

$$
H_{1}=\bigcup_{n=1}^{\infty}\left[c_{n}^{(1)}, d_{n}^{(1)}\right]
$$

and

$$
H_{1} \subset\left[a_{1}-\frac{b_{1}-a_{1}}{2}, a_{1}+\frac{b_{1}-a_{1}}{2}\right] \backslash C \subset \bigcup_{k=1}^{\infty}\left(a_{k}, b_{k}\right) .
$$

Simultaneously, $d_{1}\left(H_{1}, a_{1}\right)=1$ by $(1)$.

Since $a_{2} \notin \bigcup_{k=1}^{\infty}\left(a_{k}, b_{k}\right)$, so $a_{2} \notin H_{1} \cup\left\{a_{1}\right\}$. Put $\alpha_{2}=\operatorname{dist}\left(a_{2}, H_{1} \cup\left\{a_{1}\right\}\right)$. The set $H_{1} \cup\left\{a_{1}\right\}$ is closed and bounded, so $\alpha_{2}>0$.

Let

$$
r_{2}=\frac{1}{2} \min \left(b_{2}-a_{2}, \alpha_{2}\right) \text {. }
$$

Obviously, $r_{2}>0$ and $\left(a_{2}-r_{2}, a_{2}+r_{2}\right) \cap H_{1}=\emptyset$. By Lemma 2.12 there exists a left interval set $E_{2}$ at the point $a_{2}$, which is a union of open intervals and fulfils the conditions

$$
E_{2} \subset\left[a_{2}-r_{2}, a_{2}\right], \quad C \cap\left[a_{2}-r_{2}, a_{2}\right] \subset E_{2} \cup\left\{a_{2}\right\}
$$

and

$$
d_{1}\left(E_{2}, a_{2}\right)=0
$$

Put

$$
\widetilde{H}_{2}=\left[a_{2}-r_{2}, a_{2}\right] \backslash\left(E_{2} \cup\left\{a_{2}\right\}\right) .
$$

Obviously, $\widetilde{H}_{2}$ is a left interval set at the point $a_{2}$, which is a union of closed intervals

$$
\widetilde{H}_{2}=\bigcup_{n=1}^{\infty}\left[c_{n}^{(2)}, d_{n}^{(2)}\right] \subset\left[a_{2}-r_{2}, a_{2}\right] \backslash C \subset \bigcup_{k=1}^{\infty}\left(a_{k}, b_{k}\right) .
$$

The set $2 a_{2}-\widetilde{H}_{2}$ is a right interval set at the point $a_{2}$. Put $H_{2}=\widetilde{H}_{2} \cup$ $\left(2 a_{2}-\widetilde{H}_{2}\right)$. Then $H_{2}$ is an interval set at the point $a_{2}$, symmetric with respect to $a_{2}, H_{2} \subset \bigcup_{k=1}^{\infty}\left(a_{k}, b_{k}\right)$ and $d_{1}\left(H_{2}, a_{2}\right)=1$ by $(2)$. 
Suppose now that we have defined the interval sets $H_{1}, H_{2}, \ldots, H_{m}$ at the points $a_{1}, a_{2}, \ldots, a_{m}$ respectively, each of them is a countable union of closed intervals, $H_{i} \subset \bigcup_{k=1}^{\infty}\left(a_{k}, b_{k}\right), d_{1}\left(H_{i}, a_{i}\right)=1$ and $H_{i}$ is symmetric with respect to $a_{i}, i=1, \ldots, m$. Since $a_{m+1} \notin \bigcup_{k=1}^{\infty}\left(a_{k}, b_{k}\right)$, therefore $a_{m+1} \notin$ $\bigcup_{i=1}^{m}\left(H_{i} \cup\left\{a_{i}\right\}\right)$. Put $\alpha_{m+1}=\operatorname{dist}\left(a_{m+1}, \bigcup_{i=1}^{m} H_{i}\right)$ and

$$
r_{m+1}=\frac{1}{2} \min \left(b_{m+1}-a_{m+1}, \alpha_{m+1}\right) \text {. }
$$

Obviously, $r_{m+1}>0$ and $\left(a_{m+1}-r_{m+1}, a_{m+1}+r_{m+1}\right) \cap \bigcup_{i=1}^{m} H_{i}=\emptyset$. By Lemma 2.12 there exists a left interval set $E_{m+1}$ at the point $a_{m+1}$, which is a union of open intervals and fulfils the conditions

$E_{m+1} \subset\left[a_{m+1}-r_{m+1}, a_{m+1}\right], \quad C \cap\left[a_{m+1}-r_{m+1}, a_{m+1}\right] \subset E_{m+1} \cup\left\{a_{m+1}\right\}$ and

$$
d_{1}\left(E_{m+1}, a_{m+1}\right)=0 .
$$

Put

$$
\widetilde{H}_{m+1}=\left[a_{m+1}-r_{m+1}, a_{m+1}\right] \backslash\left(E_{m+1} \cup\left\{a_{m+1}\right\}\right) .
$$

Obviously, $\widetilde{H}_{m+1}$ is a left interval set at the point $a_{m+1}$, which is a union of closed intervals

$$
\widetilde{H}_{m+1}=\bigcup_{n=1}^{\infty}\left[c_{n}^{(m+1)}, d_{n}^{(m+1)}\right] \subset\left[a_{m+1}-r_{m+1}, a_{m+1}\right] \backslash C \subset \bigcup_{k=1}^{\infty}\left(a_{k}, b_{k}\right) .
$$

The set $2 a_{m+1}-\widetilde{H}_{m+1}$ is a right interval set at the point $a_{m+1}$. Put $H_{m+1}=\widetilde{H}_{m+1} \cup\left(2 a_{m+1}-\widetilde{H}_{m+1}\right)$. Then $H_{m+1}$ is an interval set at the point $a_{m+1}$, symmetric with respect to $a_{m+1}, H_{m+1} \subset \bigcup_{k=1}^{\infty}\left(a_{k}, b_{k}\right)$ and $d_{1}\left(H_{m+1}, a_{m+1}\right)=1$ by $(3)$.

So we have defined the sequence $\left\{H_{i}\right\}_{i \in N}$ of interval sets at the points $a_{i}$, respectively, such that $H_{i}$ is symmetric with respect to $a_{i}, H_{i} \subset \bigcup_{k=1}^{\infty}\left(a_{k}, b_{k}\right)$ and $d_{1}\left(H_{i}, a_{i}\right)=1$ for $i \in \mathbb{N}$.

Define the function $\widetilde{g}_{i}$ on $H_{i} \cup C$ by

$$
\tilde{g}_{i}(t)=\left\{\begin{array}{cc}
\frac{1}{2^{i}} & \text { for } \quad t \in H_{i} \\
0 & \text { for } \quad t \in C
\end{array}\right.
$$

and extend $\widetilde{g}_{i}$ on $\mathbb{R} \backslash\left(H_{i} \cup C\right)$ in such a way that it is piecewice linear on $\mathbb{R}$ and bounded by $1 / 2^{i}$. Let

$$
g_{i}(t)= \begin{cases}\frac{1}{2^{i}} & \text { for } t=a_{i}, \\ \widetilde{g}_{i}(t) & \text { for } t \in \mathbb{R} \backslash\left\{a_{i}\right\},\end{cases}
$$

for $i \in \mathbb{N}$. Obviously $0 \leq g_{i}(t) \leq 1 / 2^{i}$ for $t \in \mathbb{R}, i \in \mathbb{N}$. Put

$$
\bar{g}_{i}(t)=g_{i}\left(t+a_{i}\right)
$$


for $i \in \mathbb{N}, t \in \mathbb{R}$, and let

$$
\bar{f}_{i}(x, y)=\bar{g}_{i}(\max (|x|,|y|)), \quad f_{i}(x, y)=\bar{f}_{i}\left(x-a_{i}, y\right)
$$

for $(x, y) \in \mathbb{R}^{2}, i \in \mathbb{N}$. Finally, define

$$
f(x, y)=\sum_{i=1}^{\infty} f_{i}(x, y)
$$

for $(x, y) \in \mathbb{R}^{2}$.

First we observe that $f$ is not in Baire* 1 class. Let $D=C \times\{0\}$. Clearly, $D$ is a perfect set. Let $U=(p, q) \times(r, s)$ be an arbitrary interval such that $D \cap U \neq \emptyset$. Then there exists $i \in \mathbb{N}$ such that $\left(a_{i}, 0\right) \in D \cap U$ (this is possible because $C^{\prime}$ is dense in $C$ ). Since $C$ is perfect, there exists a sequence $\left\{x_{n}\right\}_{n \in \mathbb{N}}$ such that $x_{n} \underset{n \rightarrow \infty}{\longrightarrow} a_{i}, x_{n} \neq a_{k}$ for $k \in \mathbb{N}$ and $x_{n} \in C \cap(p, q)$ for $n \in \mathbb{N}$. Then $f\left(x_{n}, 0\right)=0$ and $f\left(a_{i}, 0\right) \geq 1 / 2^{i}>0$, therefore $f \mid D$ is not continuous.

It remains to prove that $f:\left(\mathbb{R}^{2}, \mathcal{T}_{r}\right) \rightarrow(\mathbb{R}, \mathcal{O})$ is continuous. First, we shall show that $\bar{f}_{i}:\left(\mathbb{R}^{2}, \mathcal{T}_{r}\right) \rightarrow(\mathbb{R}, \mathcal{O})$ is continuous for $i \in \mathbb{N}$. Fix $i \in \mathbb{N}$. The functions $\widetilde{g}_{i}$ and $g_{i}$ are continuous on the real line except for a point $a_{i}$, hence $\bar{g}_{i}$ is continuous at each point of the real line except for 0 . Consequently, $\bar{f}_{i}$ is continuous with respect to the Euclidean topology at each point of the plane except for $(0,0)$, as the superposition of continuous functions. We have $d_{1}\left(H_{i}, a_{i}\right)=1, H_{i}$ is symmetric with respect to $a_{i}$ and $g_{i}(t)=1 / 2^{i}$ for $t \in H_{i} \cup\left\{a_{i}\right\}$, so $\bar{f}_{i}$ is constant on the set $E=\{(x, y) \in$ $\mathbb{R}^{2}: \max (|x|,|y|) \in$ int $\left.H_{i}-a_{i}\right\}$. From Corollary 2.7 it follows that $d_{r}(E,(0,0))=1$, so $\bar{f}_{i}$ is continuous at the point $(0,0)$ with respect to the topology $\mathcal{T}_{r}$. Thus for each $i \in \mathbb{N}$ the function $f_{i}$ is continuous with respect to the topology $\mathcal{T}_{r}$ on the domain. Consequently, $f$ is continuous with respect to $\mathcal{T}_{r}$ as a sum of uniformly convergent series of functions continuous with respect to $\mathcal{T}_{r}$.

\section{The category density case}

Denote by $\mathcal{B}_{k}$ the family of all sets having the Baire property and by $I_{k}$ - the family of all sets of the first category on $\mathbb{R}^{k}, k=1,2$.

If a point $p \in \mathbb{R}^{k}$ is the $I_{k}$-ordinary density point ( $I_{k}$-ordinary dispersion point) of a set $A \in \mathcal{B}_{k}$, then we shall write $d_{I_{k}}(A, p)=1\left(d_{I_{k}}(A, p)=0\right), k=$ 1,2 . If a plane set is contained in a real line, then we shall consider its linear $I$-density $d_{I_{1}}$.

We shall say that two sets $A, B \subset \mathbb{R}^{2}$ are equivalent $(A \sim B)$ if and only if $A \triangle B \in I_{2}$. Let $\mathcal{D}_{I_{1}}\left(\mathcal{D}_{I_{2}}\right)$ denote the $I$-density topology on the real line (the ordinary $I$-density topology on the plane). See [1]. 
If $A \subset \mathbb{R}$ then $n \cdot A=\{n \cdot a: a \in A\}$. If $A \subset \mathbb{R}^{2}$ then $(n, n) \cdot A=$ $\{(n \cdot x, n \cdot y):(x, y) \in A\}$.

Definition 3.1. We say that a point $\left(x_{0}, y_{0}\right) \in \mathbb{R}^{2}$ is a regular $I$-density point of a set $A \subset \mathbb{R}^{2}$ if and only if there exists a set having the Baire property $B \subset A$ such that $d_{I_{2}}\left(B,\left(x_{0}, y_{0}\right)\right)=1$ and $B \in \mathcal{R}\left(x_{0}, y_{0}\right)$. In this case we write $d_{r I}\left(A,\left(x_{0}, y_{0}\right)\right)=1$.

For $A \in \mathcal{B}_{2}$ let

$$
\Phi_{I}(A)=\left\{(x, y) \in \mathbb{R}^{2}: d_{I_{2}}(A,(x, y))=1\right\}
$$

and

$$
\Phi_{r I}(A)=\left\{(x, y) \in \mathbb{R}^{2}: d_{r I}(A,(x, y))=1\right\} .
$$

Obviously, $\Phi_{r I}(A) \subset \Phi_{I}(A)$ for $A \in \mathcal{B}_{2}$ and the operator $\Phi_{r I}$ has properties analogous to those for $\Phi_{r}$ considered in measure case, i.e.

$1^{0} \Phi_{r I}(\emptyset)=\emptyset, \Phi_{r I}\left(\mathbb{R}^{2}\right)=\mathbb{R}$

$2^{0}$ if $A \subset B$, then $\Phi_{r I}(A) \subset \Phi_{r I}(B)$;

$3^{0} \Phi_{r I}(A \cap B)=\Phi_{r I}(A) \cap \Phi_{r I}(B)$

for $A, B \in \mathcal{B}_{2}$. The set $A=[-1,1] \times((\mathbb{R} \backslash \mathbb{Q}) \cap[-1,1])$ is of the second category on the plane and $\Phi_{r I}(A)=\emptyset$, so $A \triangle \Phi_{r I}(A) \notin \mathcal{I}_{2}$ and the theorem analogous to the Lebesgue Density Theorem for $\Phi_{r I}$ does not hold and neither does the equality of values of $\Phi_{r I}$ for equivalent sets.

Put

$$
\mathcal{T}_{r I}=\left\{A \in \mathcal{B}_{2}: A \subset \Phi_{r I}(A)\right\}
$$

Theorem 3.2. The family $\mathcal{T}_{r I}$ is a topology on the plane, essentially stronger than the Euclidean topology and essentially weaker than the I-density topology $\mathcal{D}_{I_{2}}$ on the plane.

The proof is analogous to the proof of Theorem 2.2.

Lemma 3.3. Let $B \in \mathcal{R}$. The set $B$ has the Baire property $\left(B \in \mathcal{B}_{2}\right)$ if and only if $B_{[R]} \in \mathcal{B}_{1}$.

The proof based upon the Kuratowski-Ulam Theorem (see [6, Theorems 15.1 and 15.2]) is analogous to the proof of Lemma 2.3.

Definition 3.4. We say that $x \in \mathbb{R}$ is an inner $I$-density point of $A \subset \mathbb{R}$ if and only if there exists a set $B \in \mathcal{B}_{1}$ such that $B \subset A$ and $d_{I_{1}}(B, x)=1$.

Theorem 3.5. If $(0,0)$ is a regular $I$-density point of a set $A \subset \mathbb{R}^{2}$ then $(0,0)$ is an inner (linear) I-density point of $A \cap p_{0}$ and $A \cap p^{0}$. 
Proof. We shall show that if $d_{r I}(A,(0,0))=1$ then $(0,0)$ is an inner $I$ density point of $A \cap p_{0}$.

By our assumption there exists a set $B \in \mathcal{B}_{2} \cap \mathcal{R}, B \subset A$ such that $d_{I_{2}}(B,(0,0))=1$. By the definition of an $I_{2}$-density point, for every increasing sequence $\left\{n_{m}\right\}_{m \in \mathbb{N}}$ of natural numbers there exists a subsequence $\left\{n_{m_{p}}\right\}_{p \in \mathbb{N}}$ of $\left\{n_{m}\right\}_{m \in \mathbb{N}}$ such that

$$
\limsup _{p}\left(n_{m_{p}}, n_{m_{p}}\right) \cdot B^{\prime} \cap[-1,1]^{2} \in I_{2} .
$$

Suppose that $d_{I_{1}}\left(B_{[R]}, 0\right) \neq 1$. Then there exists an increasing sequence $\left\{n_{m}\right\}_{m \in \mathbb{N}}$ of natural numbers such that for every subsequence $\left\{n_{m_{p}}\right\}_{p \in \mathbb{N}}$ of $\left\{n_{m}\right\}_{m \in \mathbb{N}}$ we have

$$
\underset{p}{\limsup } n_{m_{p}} \cdot\left(B_{[R]}\right)^{\prime} \cap[-1,1]=\bigcap_{k=1}^{\infty} \bigcup_{p=k}^{\infty} n_{m_{p}} \cdot\left(B_{[R]}\right)^{\prime} \cap[-1,1] \notin I_{1} .
$$

Note that if $x \in \underset{p}{\limsup } n_{m_{p}} \cdot\left(B_{[R]}\right)^{\prime} \cap[-1,1]$ then

$$
(\{x\} \times(-x, x)) \subset \bigcap_{k=1}^{\infty} \bigcup_{p=k}^{\infty}\left(n_{m_{p}}, n_{m_{p}}\right) \cdot B^{\prime} \cap[-1,1]^{2},
$$

so the set

$$
\left[\bigcap_{k=1}^{\infty} \bigcup_{p=k}^{\infty}\left(n_{m_{p}}, n_{m_{p}}\right) \cdot B^{\prime} \cap[-1,1]^{2}\right]_{x}
$$

is of the second category on the line for all $x \in \lim \sup _{p} n_{m_{p}} \cdot\left(B_{[R]}\right)^{\prime} \cap[-1,1]$, which together with (4) gives a contradiction with the Kuratowski-Ulam theorem. Analogously for $A \cap p^{0}$.

Theorem 3.6. Let $B \in \mathcal{R}$. If $d_{I_{1}}\left(B_{[R]}, 0\right)=1$ then $d_{I_{2}}(B,(0,0))=1$.

Proof. By our assumption, for every increasing sequence $\left\{n_{m}\right\}_{m \in \mathbb{N}}$ of natural numbers there exists a subsequence $\left\{n_{m_{p}}\right\}_{p \in \mathbb{N}}$ of $\left\{n_{m}\right\}_{m \in \mathbb{N}}$ such that

$$
\underset{p}{\limsup } n_{m_{p}} \cdot\left(B_{[R]}\right)^{\prime} \cap[-1,1] \in I_{1} .
$$

Using the notation from the proof of Lemma 2.3 we observe that

$$
\begin{aligned}
& {\left[\left(\limsup _{p} n_{m_{p}} \cdot\left(B_{[R]}\right)^{\prime} \cap[-1,1]\right) \times[-1,1]\right] \cap C_{I}} \\
& \left.=\underset{p}{(\limsup }\left(n_{m_{p}}, n_{m_{p}}\right) \cdot B^{\prime}\right) \cap[-1,1]^{2} \cap C_{I} .
\end{aligned}
$$

By Theorem 15.3 in [6] we have

$$
\left(\limsup _{p} n_{m_{p}} \cdot\left(B_{[R]}\right)^{\prime} \cap[-1,1]\right) \times[-1,1] \in I_{2}
$$


hence

$$
\left[\left(\limsup n_{p} n_{m_{p}} \cdot\left(B_{[R]}\right)^{\prime} \cap[-1,1]\right) \times[-1,1]\right] \cap C_{I} \in I_{2}
$$

and

$$
\left(\limsup _{p}\left(n_{m_{p}}, n_{m_{p}}\right) \cdot B^{\prime}\right) \cap[-1,1]^{2} \cap C_{I} \in I_{2} .
$$

As similar arguments apply to the set $C_{I I}$, we have

$$
\begin{aligned}
& \left(\limsup _{p}\left(n_{m_{p}}, n_{m_{p}}\right) \cdot B^{\prime}\right) \cap[-1,1]^{2} \\
& =\left[\left(\limsup _{p}\left(n_{m_{p}}, n_{m_{p}}\right) \cdot B^{\prime}\right) \cap[-1,1]^{2} \cap C_{I}\right] \\
& \cup\left[\left(\limsup _{p}\left(n_{m_{p}}, n_{m_{p}}\right) \cdot B^{\prime}\right) \cap[-1,1]^{2} \cap C_{I I}\right] \in I_{2} .
\end{aligned}
$$

Corollary 3.7. If $B \in \mathcal{R}$ then the following conditions are equivalent:

a) $d_{I_{1}}\left(B_{[R]}, 0\right)=1$,

b) $d_{I_{2}}(B,(0,0))=1$,

c) $d_{r I}(B,(0,0))=1$.

The same results can be obtained for any point $\left(x_{0}, y_{0}\right)$.

Let $\mathcal{D}_{I_{1}} \times \mathcal{D}_{I_{1}}$ denote the product $I$-density topology on the plane.

Theorem 3.8. $\mathcal{T}_{r I} \varsubsetneqq \mathcal{D}_{I_{1}} \times \mathcal{D}_{I_{1}}$.

The proof is analogous to the proof of Theorem 2.8 .

Theorem 3.9. Let $A \in \mathcal{B}_{2}$. If $\operatorname{proj}_{x} A \in I_{1}$ and $\operatorname{proj}_{y} A \in I_{1}$ then $A$ is closed in the topology $\mathcal{T}_{\text {rI }}$.

The proof is analogous to the proof of Theorem 2.9 and is based on the fact that if

$$
Z=\operatorname{proj}_{x} A \cup \operatorname{proj}_{y} A \cup\left(-\operatorname{proj}_{x} A\right) \cup\left(-\operatorname{proj}_{y} A\right) \in I_{1}
$$

then $\mathbb{R} \backslash Z$ is a residual set, hence $d_{I_{1}}(\mathbb{R} \backslash Z, 0)=1$.

Corollary 3.10. The space $\left(\mathbb{R}^{2}, \mathcal{T}_{r I}\right)$ is not separable.

Corollary 3.11. The space $\left(\mathbb{R}^{2}, \mathcal{T}_{r I}\right)$ is not compact. 
Considering the separation axioms we see $\mathcal{T}_{r I}$ is a Hausdorff $\left(T_{2}\right)$ topology but it is not regular $\left(T_{3}\right)$. Indeed, let $F=(\mathbb{Q} \times \mathbb{Q}) \backslash\{(0,0)\}$. Since $F$ is countable, it is closed in the topology $\mathcal{T}_{r I}$. We cannot separate this set from the point $(0,0)$ in $\mathcal{T}_{r I}$ because we cannot separate the set $\mathbb{Q} \backslash\{0\}$ from the point 0 in the $I$-density topology on the line (Theorem 3.5).

Since $\mathcal{T}_{r I}$ topology is weaker than $I$-density topology on the plane, so each function continuous with respect to $\mathcal{T}_{r I}$ on the domain is $I$-approximately continuous and, consequently, it is in the first class of Baire.

Lemma 2.12 is also true for $I$-density, and the construction of the function from Theorem 2.13 works in the category case. Thus we have the following theorem.

Theorem 3.12. There exists a function $f: \mathbb{R}^{2} \rightarrow \mathbb{R}$ continuous with respect to the topology $\mathcal{T}_{r I}$ in the domain, which is not in Baire* 1 class.

\section{References}

[1] Carrese, R., Wilczyński, W., I-density points of plane sets, Ricerche Mat. 34(1) (1985), 147-157.

[2] Cesari, L., Surface Area, Princeton University Press, Princeton, 1956.

[3] Ciesielski, K., Larson, L., Ostaszewski, K., I-density continuous functions, Mem. Amer. Math. Soc. 107(515) (1994).

[4] Goffman, C., Neugebauer, C., Nishiura, T., Density topology and approximate continuity, Duke Math. J. 28 (1961), 497-505.

[5] O’Malley, R. J., Baire*1 Darboux functions, Proc. Amer. Math. Soc. 60 (1976), 187-192.

[6] Oxtoby, J. C., Measure and Category, Springer Verlag, New York, 1971.

[7] Poreda, W., Wagner-Bojakowska, E., Wilczyński, W., A category analogue of the density topology, Fund. Math. 125 (1985), 167-173.

[8] Poreda, W., Wagner-Bojakowska, E., Wilczyński, W., Remarks on I-density and I-approximately continuous functions, Comment. Math. Univ. Carolin. 26(3) (1985), 553-563.

[9] Wilczyński, W., A generalization of density topology, Real Anal. Exchange 8(1) (1982-83), 16-20.

GraŻyna HorbaCZEWSKA

FACULTY OF MATHEMATICS

ŁÓDŹ UNIVERSITY

STEFAna Banacha 22

90-238 ŁÓDŹ

POLAND
ElżBieta WagneR-Bojakowska

FaCUlTy of Mathematics

ŁÓDŹ UNIVERSITY

Stefana Banacha 22

90-238 ŁÓDŹ

POLAND 